\title{
Optimization of Petroleum Reservoir Waterflooding Using Receding Horizon Approach
}

\author{
A. S. Grema and Y. Cao \\ School of Engineering, Cranfield University \\ Cranfield, Bedford, U.K.
}

\begin{abstract}
In this paper, static and dynamic optimization of a reservoir waterflooding process for enhanced oil recovery was studied. The dynamic optimization was achieved using receding horizon (RH) algorithms. Two forms of RH which are movingend and fixed-end RH were formulated and compared. MATLAB Reservoir Simulator (MRST) from SINTEF was used for reservoir simulation. The objective function to be maximized is net present value (NPV) of the venture while the control variable is water injection rate. Sequential quadratic programming (SQP) was applied for the optimization. It was found out that fixed-end RH gave the highest NPV with improvements of $0.81 \%$ and $1.49 \%$ over static and moving-end RH strategies respectively.
\end{abstract}

Keywords - dynamic optimization; static optimization; movingend receding horizon; fixed-end receding horizon; waterflooding process, enhanced oil recovery

\section{INTRODUCTION}

Oil and gas demand is increasing globally due to the increase in population [1]. This is springing an urgent need to recover as much oil and gas as possible in an efficient manner. Petroleum reservoirs are underground formations of porous rocks containing hydrocarbons trapped in the pores. At the initial stage of production, the reservoir pressure may be adequate to push the fluid to the surface. However, as the reservoir is depleted of its contents, its pressure declines and production decreases over time [2]. As the natural pressure of the reservoir becomes insufficient to sustain production, the common practice in the oil and gas industries is to inject water to maintain the pressure. This is called waterflooding. Sadly, only about one-third of the original oil in place (OOIP) is able to be recovered using current techniques.

Many waterflooded wells suffer from high water cut and premature water breakthrough. This occurs as the injected water meanders through conductive fractures and high permeability zones into the production wells with the oil being by-passed. This reduces the sweep efficiency greatly and as a result the ultimate recovery is drastically lowered. There are many suggested solutions to the problem of poor sweep efficiency which include mechanical isolation or squeeze cementing, use of polymeric materials [3] and employing smart production and injection wells [4]. Fluid flow into various zones of reservoir is directly proportional to the injection rate and pressure. Therefore, by efficiently controlling the injection rates and pressures, sweeping of oil can also be improved.
Smart wells have been found useful for this purpose. It provided the opportunity to improve the sweeping efficiency by imposing a suitable flow or pressure profile along the injection wells [4]. A smart well is an unconventional well equipped down hole with inflow control valves (ICVs). The ICVs divide the well into segments to provide control of flow rates, temperatures and pressures in each of these segments. The benefit of smart wells is made possible by redistributing production among the available branches which could delay or avoid water break-through as long as possible [5].

Most of the studies conducted in earlier days dealt with a simplified system. Reference [6] considered two vertical injectors and a single producer which was later followed by a study consisting of two vertical producers and a natural aquifer [7]. In the work of [8] and [9], well location, well type and well flow rates were optimized for a water flooding operation. Reference [10] carried out their optimization studies considering the extreme of well control, that is either fully opened or closed (Bang-Bang control approach) when water break-through is experienced. Reference [11] used a conjugate gradient optimization technique to maximize production rate using smart wells. But [12] used optimal control algorithms to maximize recovery or net present value (NPV) of a waterflooding process over a period of time. Both purely pressure and purely flow rate constrained scenarios were considered. Reference [13] used a combination of ensemble Kalman filter technique for model updating and automated adjoint-based method for waterflood optimization. In the work of [14], the optimization was performed using adjoint-based method but under nonlinear constraints. One shortcoming of adjoint-based technique is that it requires a detailed knowledge of the reservoir simulator. However, [15] used ensemble Kalman filter (EnKF) as the optimization algorithms to overcome the shortcoming of adjoint-based method. Reference [16] investigated the effect of formulation and initial guess on two gradient based methods, steepest descent and conjugate gradient. Reference [2] optimized the operation of a smart well during waterflooding using an Explicit Singly Diagonally Implicit Runge-Kutta (ESDIRK) method and a quasi-Newton Sequential Quadratic Programming (SQP) in the optimal control strategy. Reference [17] extended the work of [2] by including gradient computation based on continuous-time adjoint equation. In this study, optimization of reservoir waterflooding was carried 
out using receding horizon strategy. Two approaches of receding horizon namely fixed-end and moving-end horizon were investigated and compared against a static optimization strategy. The paper is organized as follows; section II discusses the problem formulation followed by results and discussion in section III. Finally, conclusions and recommendations for future work are highlighted in section IV.

\section{PROBLEM FORMULATION}

\section{A. Reservoir Model and Dynamics}

A simple homogenous reservoir as shown in Fig. 1 with one horizontal producer and a vertical injector was considered. The wells were arbitrarily located in the reservoir. The injector well has five perforations and each perforation was modeled separately so that it can be controlled independently. The reservoir is $20 \times 20 \times 5 \mathrm{~m}^{3}$. A Cartesian gridding system was used to describe the reservoir where each cell has dimensions of $1 \times 1 \times 1 \mathrm{~m}^{3}$. The reservoir has a porosity of $30 \%$ and permeability of $100 \mathrm{mD}$ with two-phase oil-water system. The initial water saturation was taken to be 0.1 . This implies that OOIP is $540 \mathrm{~m}^{3}$. The considered system is extremely simplified to make the concept clear but as demonstrated by [18], it is still viable because this kind of system can be found as isolated segments of a real reservoir. MATLAB Reservoir Simulator Toolbox (MRST) from SINTEF was used for the simulation.

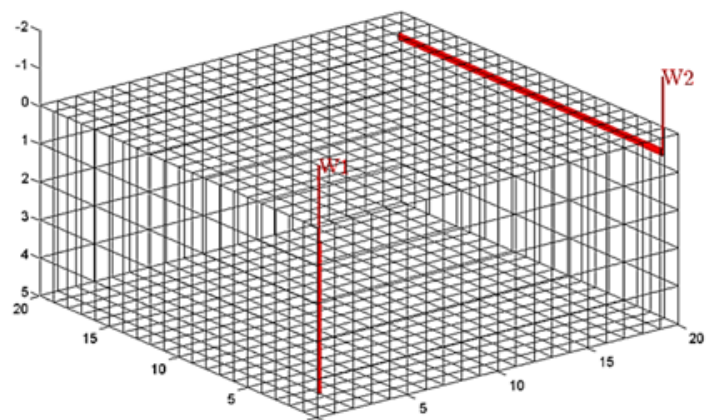

Figure 1: Reservoir Geometry with Wells Configuration (modified from SINTEF Examples)

Reservoir model was presented in a compact and discretized form by [19] as

$$
g_{k+1}\left(u_{k+1}, x_{k}, x_{k+1}\right)=0, k=0, \ldots K-1
$$

Where $g$ is a nonlinear vector-valued function, $u$ is the input vector (or control vector) such as water injection rate and/or production rate, $x$ is the state vector which include reservoir pressure, and oil and water saturation. The subscript k indicates discrete time and $\mathrm{K}$ is end time. For the model to be complete, initial conditions need to be specified which is

$$
x_{0}=\check{x}_{0}
$$

The outputs which are usually well production rates are combined in an output vector $\mathrm{y}$ as functions of state variables $\mathrm{x}$ and input variables $u$.

$$
y_{k+1}=h\left(u_{k+1}, x_{k+1}\right)
$$

\section{B. Receding Horizon Control Strategy}

Optimal control for instance in [20], and [2] was used to find the values of input variables $u$ that maximize or minimize a cost function $J$. For the fact that $J$ is a function of $x$, which in turn is a function of $\mathrm{u}$, the influence of $\mathrm{u}$ on $\mathrm{J}$ cannot be directly determined but the changes in $\mathrm{x}$ need to be determined first.

As mentioned by [21], receding horizon control (RHC) is a very popular extension of optimal control algorithms that has been developed for both linear and nonlinear systems. It involves solving a fixed horizon optimization problem where a sequence of predicted inputs is determined over a prediction horizon (for instance T time steps) and then implementing only the first step in the series. The prediction time is moved one step forward and the whole process is repeated [22]. Two strategies of RHC exist with respect to the nature of the prediction horizon. Fixed-end RHC where the prediction horizon is fixed to the production period and decreases subsequently as the prediction time advances. The second strategy is moving-end RHC in which the prediction period is a fixed time period which does not change throughout the optimization process. These strategies are adopted in this work for the aim of optimization which can be viewed pictorially as in Fig. 2 where the total production time is divided into $\mathrm{n}$ equal sampling time with $\mathrm{T}_{\mathrm{p}}$ being the prediction time.
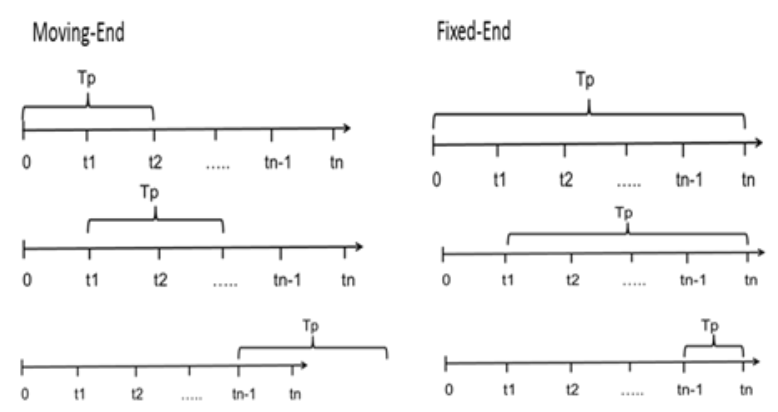

Figure 2: Receding Horizon Strategies

\section{Approach}

Three optimization strategies were considered. The first being static optimization where an optimal injection rate was found for the entire production period. However, this is not often feasible in practice because it may require unrealistic bottomhole pressures [12]. For this reason dynamic optimization was performed using the concept of receding horizon ( $\mathrm{RH})$. The objective function used is maximization of 
net present value (NPV) of the waterflooding process given by [23] as in (4)

$$
J_{k}=\left\{\frac{\sum_{i=1}^{N_{i n j}} r_{w i}\left(u_{w i, i}\right)_{k}+\sum_{j=1}^{N_{p r o d}}\left[r_{w p}\left(y_{w p, j}\right)_{k}+r_{o}\left(y_{o, j}\right)_{k}\right]}{(1+b)^{\frac{t_{k}}{\tau}}}\right\} \Delta t_{k}
$$

Where $r_{o}$ is the oil price taken as $+\$ 80 / \mathrm{m}^{3}$, while both water injection cost, $r_{w i}$ and production cost, $r_{w p}$ were taken as $\$ 5 / \mathrm{m}^{3}, \mathrm{u}_{\mathrm{wi}}, \mathrm{y}_{\mathrm{wp}}$ and $\mathrm{y}_{\mathrm{o}}$ are water injection, water and oil production rates respectively. The number of injection and production wells is given by $\mathrm{N}_{\text {inj }}$ and $\mathrm{N}_{\text {prod }}$ respectively. The discount factor is $\mathrm{b}$ per unit time $\tau$ [s] and was assumed to be $10 \%$ per year; $t_{k}[s]$ is the time at time step $\mathrm{k}$ while $\Delta \mathrm{t}_{\mathrm{k}}$ is the time interval between successive steps. The manipulated variable used is water injection rate which is bounded in the range $[0.0110] \mathrm{m}^{3} /$ day.

For the three optimization strategies, two-year production period was used; and two months sampling period was applied for RH strategies. Using these settings for fixed-end $\mathrm{RH}$, optimization was first performed for two years and the optimal injection rate found is implemented for two months. The current (after two-month production) reservoir state is used as an initial state for a 22-month optimization with the optimum rate found implemented for another two-month production. The procedure continues to the end of the production period. In the case of moving-end $\mathrm{RH}$, the prediction horizon is constant one year with two months sampling period. This means optimization is carried out for one year and the optimum injection rate is applied for two month production and the current reservoir state is used for another one year optimization and so on till the end of the production period.

\section{RESULTS AND DISCUSSIONS}

With the boundary constraints set on the injection rates, optimum values for the three strategies are shown in Figs. 3, 4 and 5, respectively. The injection rates are for the individual perforations with perf1 being the topmost while perf5 the lowest perforations. It can be seen that for static optimization case all the perforations were shut down for the entire production period with the exception of perf5 whose optimum injection rate was found to be $0.82 \mathrm{~m}^{3} /$ day. Actually, the other four perforations were set to the lower boundary constraint by the optimization algorithm (Fig. 3).

For the case of RH approaches, the injection rates were continuously being distributed among the perforations as the production progresses. A higher injection rate was initially allocated to the lowest perforation for these strategies. In moving-end RH approach (Fig. 4), an extremely high injection rate was found at the beginning of production period which was subsequently reduced to a maximum of about $0.5 \mathrm{~m}^{3} /$ day after a year of production. This is the reason of an initial accelerated production as can be observed in Fig. 7. Both high oil and water productions are initially recorded as well as their cumulative production (Fig. 8). This point can also be further confirmed by observing the distribution of water saturation in the reservoir in Fig. 9 in which higher saturations are seen after 120 and 365 days in comparison to the case of fixed-end $\mathrm{RH}$ in Fig. 10. The early accelerated production of this strategy is also evidenced from high NPV recorded at the early production life (Fig. 6).

A longer plateau period of oil production can be seen due to average injection rates of about $1.0 \mathrm{~m}^{3} /$ day in the case of fixedend RH (Figs. 5 and 7).

However, early production rate is much lower for this strategy (fixed-end $\mathrm{RH}$ ) than moving-end $\mathrm{RH}$, the effect was counteracted by a lower decline rate. The result is revealed by a steady rise in NPV which was initially lagging behind movingend RH but surpassed it after 1.4 year (Fig. 6).

Table 1 compares the effectiveness of the three methods in terms of economic benefits of the venture. Fixed-end RH gave the highest NPV followed by static approach after two years of production. The relative increase in NPV of the former over the latter is $0.81 \%$ while $1.49 \%$ increment was realized with respect to moving-end $\mathrm{RH}$. Although, the relative difference in NPV of the three approaches is not that high, the relative increment will be apparent when the techniques are applied to a larger and real reservoir. The low NPV recorded for the case of moving-end $\mathrm{RH}$ is due to a drastic cut in water injection after a year production. Again, this method may favor reservoir production with high heterogeneities

\section{CONCLUSIONS AND RECOMMENDATIONS}

Static and dynamic optimizations of reservoir waterflooding were carried out. We have considered two forms of RH strategy for the dynamic optimization. For all the cases considered, the highest NPV was recorded using fixed-end RH method with an increase of $0.81 \%$ and $1.49 \%$ over static and moving-end strategies respectively. This was made possible by distributing moderate water injection rates to the five perforations right from the beginning to the end of the slated production period for the fixed-end RH. The least NPV recorded in the case of moving-end $\mathrm{RH}$ was due to excessive cut of water injection after a year of production which affected the oil rate and hence the NPV. It is believed that this method (moving-end $\mathrm{RH}$ ) may favor high NPV than the other two if applied to a real reservoir with high heterogeneities because it is associated with more frequent model updating.

The effectiveness of SQP which is a local optimizer depends on a good starting point to prevent being trapped in a local optimum. For this reason different starting points should be tested for the optimization strategies. Some global optimization techniques should also be used so as to get a global optimum at each stage of the horizon for $\mathrm{RH}$ approaches.

\section{ACKNOWLEDGMENT}

We are grateful to SINTEF for providing a free license of MRST software and for modifying one of the accompanied examples. The effort of Petroleum Technology Development 
Fund (PTDF), Nigeria for the financial support rendered is also acknowledged.
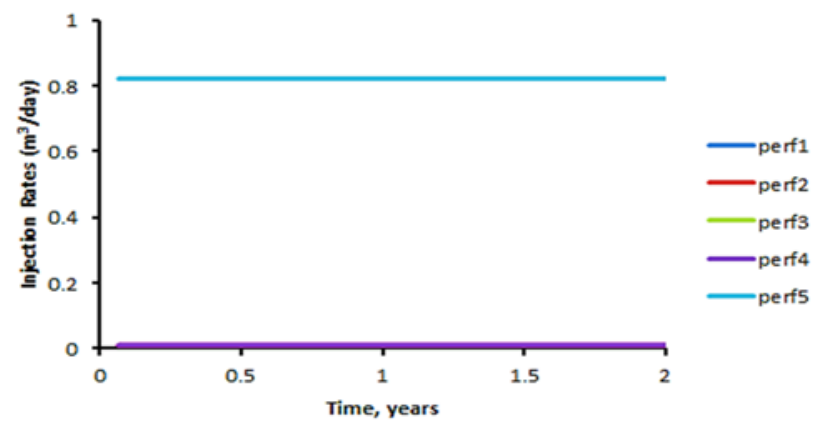

Figure 3: Injection Rates for Static Optimization

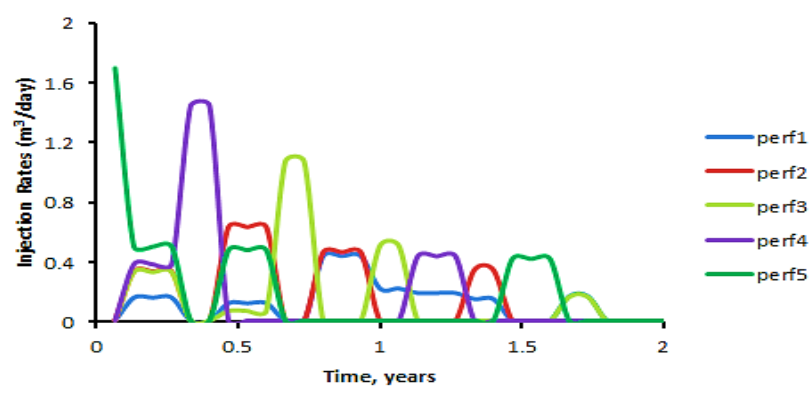

Figure 4: Injection Rates for Moving-End RH

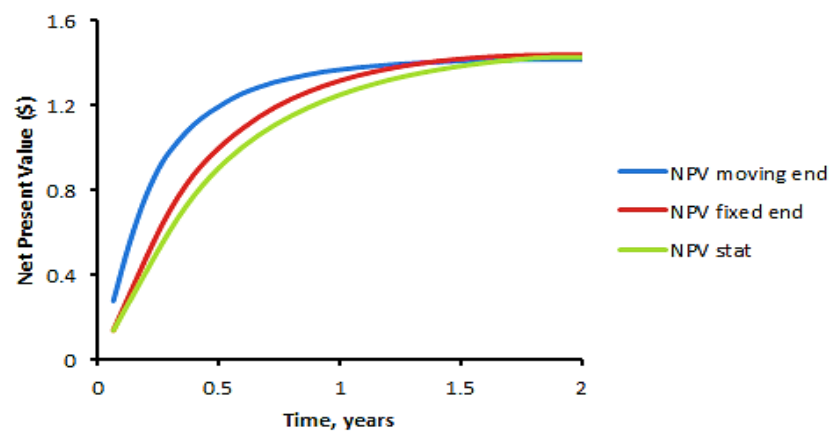

Figure 6: NPV for the Three Optimization Cases

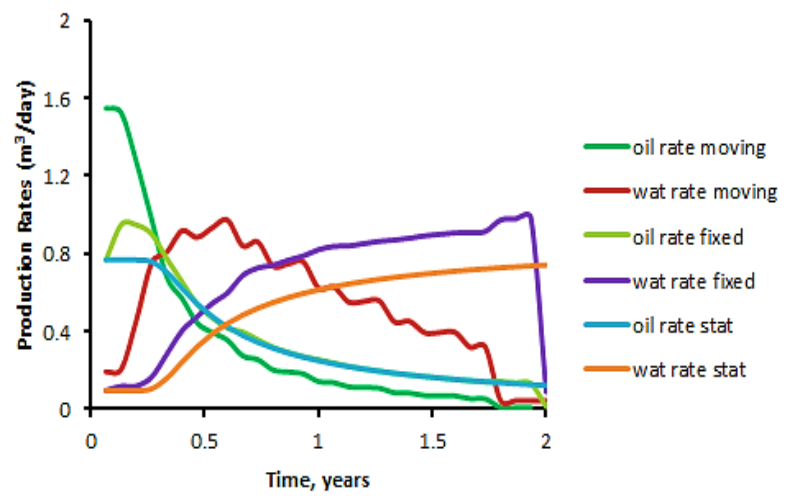

Figure 7: Production Rates for the Three Optimization Cases

TABLE 1: Comparison of Optimization Strategies

\begin{tabular}{|c|c|c|c|c|}
\hline Strategy & NPV (\$) & $\begin{array}{l}\text { Cumulative Oil } \\
\text { Production }\left(\mathbf{m}^{3}\right)\end{array}$ & $\begin{array}{c}\text { Cumulative Water } \\
\text { Production }\left(\mathrm{m}^{3}\right)\end{array}$ & $\begin{array}{c}\text { NPV Relative } \\
\text { Decrement (\%) }\end{array}$ \\
\hline Static Optimization & 14,278 & 245.48 & 373.22 & 0.81 \\
\hline Fixed-End RH & 14,393 & 260.22 & 485.61 & - \\
\hline Moving-End RH & 14,181 & 242.61 & 385.23 & 1.49 \\
\hline
\end{tabular}

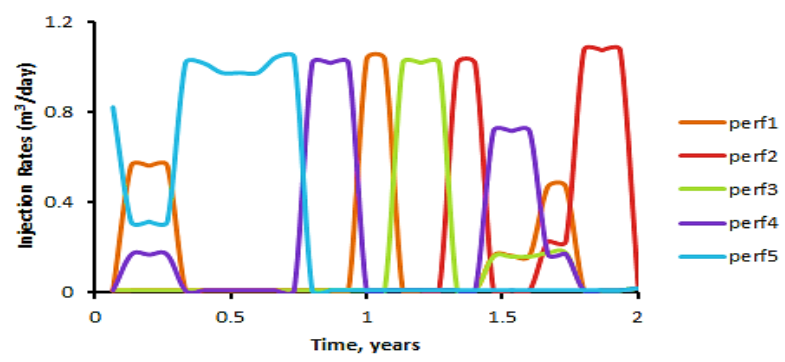

Figure 5: Injection Rates for Fixed-End RH

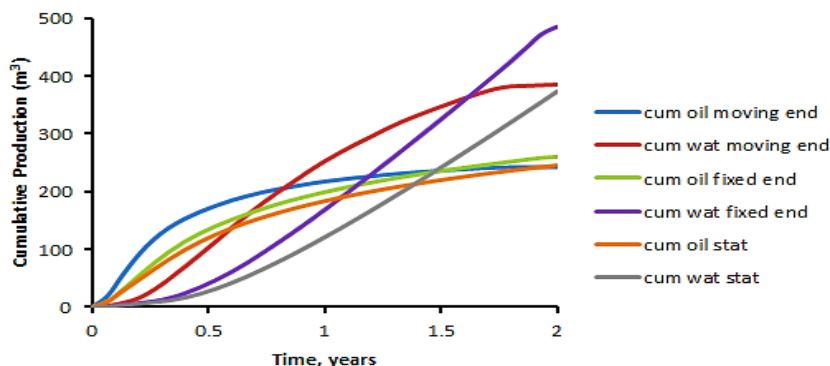

Figure 8: Total Production for the Three Optimization Cases 


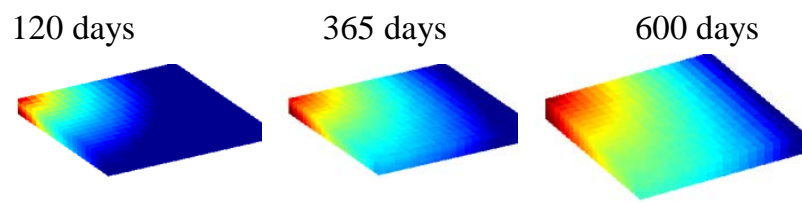

Fig.9: Water Saturation Distribution for Moving-End RH
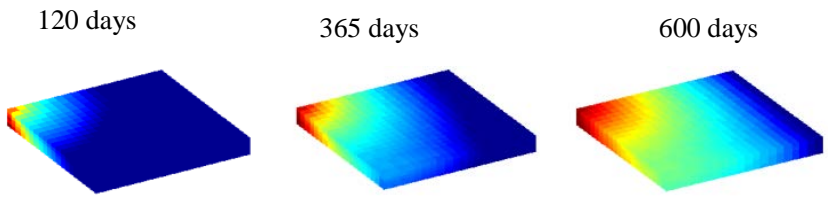

Fig.10: Water Saturation Distribution for Fixed-End RH

\section{REFERENCES}

[1] ExxonMobil (2012), The Outlook for Energy: A View to 2040, available at:

www.exxonmobil.com/Corporate/files/news_pub_eo.pdf (accessed 07/2012).

[2] Völcker, C., Jorgensen, J. B. and Stenby, E. H. (2011), "Oil Reservoir Production Optimization using Optimal Control", 2011 50th IEEE Conference on Decision and Control and European Control Conference, CDC-ECC 2011, 12 December 2011 through 15 December 2011, Orlando, FL, pp. 7937.

[3] Mody, B. G. and Dabbous, M. K. (1989), "Reservoir Sweep Improvement with Cross-Linked Polymer Treatments", Middle East Oil Show, 11-14 March 1989, 1989 Copyright 1989, Society of Petroleum Engineers, Bahrain.

[4] Brouwer, D. R., Jansen, J. D., van der Starre, S., van Kruijsdijk, C. P. J. W. and Berentsen, C. W. J. (2001), "Recovery Increase through Water Flooding with Smart Well Technology", SPE European Formation Damage Conference, 21-22 May 2001, Copyright 2001, Society of Petroleum Engineers Inc., The Hague, Netherlands.

[5] Meum, P., Tøndel, P., Godhavn, J. and Aamo, O. M. (2008), "Optimization of Smart Well Production Through Nonlinear Model Predictive Control", Intelligent Energy Conference and Exhibition, 25-27 February 2008, Society of Petroleum Engineers, Amsterdam, The Netherlands.

[6] Asheim, H. (1987), "Optimal Control of Water Drive", 01 Jan 1987, Society of Petroleum Engineers.
[7] Asheim, H. (1988), "Maximization of Water Sweep Efficiency by Controlling Production and Injection Rates", European Petroleum Conference, 16-19 October 1988, 1988 Copyright 1988, Society of Petroleum Engineers, London, United Kingdom.

[8] Virnovsky, G. A. (1988), "On Optimal Control of Multiphase Porous Flow in an oil Bed", Comput. Math. Math. Phys., vol. 28, no. 3, pp. 156-163.

[9] Virnovsky, G. A. (1991), "Water Flooding Strategy Design Using Optimal Control Theory", The Sixth European IOR Symposium, 21-23 May, Stavanger, Norway.

[10] Sudaryanto, B. and Yortsos, Y. C. (2000), "Optimization of Fluid Front Dynamics in Porous Media Using Rate Control. I. Equal Mobility Fluids", Physics of Fluids, vol. 12, no. 7, pp. 1656-1670.

[11] Yeten, B., Durlofsky, L. J. and Aziz, K. (2003), "Optimization of Nonconventional Well Type, Location, and Trajectory", vol. 8, no. 3, pp. 200-200-210.

[12] Brouwer, D. R. and Jansen, J. -. (2004), "Dynamic Optimization of Waterflooding With Smart Wells Using Optimal Control Theory", SPE Journal, vol. 9, no. 4, pp. 391-391-402.

[13] Brouwer, D. R., NÃ'vdal, G., Jansen, J. D., Vefring, E. H. and van Kruijsdijk, C. P. J. W. (2004), "Improved Reservoir Management Through Optimal Control and Continuous Model Updating", SPE Annual Technical Conference and Exhibition, 26-29 September 2004, Society of Petroleum Engineers, Houston, Texas.

[14] Sarma, P., Chen, W. H., Durlofsky, L. J. and Aziz, K. (2008), "Production Optimization With Adjoint Models Under Nonlinear Control-State Path Inequality Constraints", vol. 11, no. 2, pp. 326-326-339.

[15] Lorentzen, R. J., Berg, A., Naevdal, G. and Vefring, E. H. (2006), "A New Approach for Dynamic Optimization of Waterflooding Problems", Intelligent Energy Conference and Exhibition, 11-13 April 2006, Society of Petroleum Engineers, Amsterdam, The Netherlands.

[16] Asadollahi, M. and Naevdal, G. (2009), "Waterflooding Optimization Using Gradient Based Methods", SPE/EAGE Reservoir Characterization and Simulation Conference, 19-21 October 2009, Society of Petroleum Engineers, Abu Dhabi, UAE.

[17] Capolei, A., Volcker, C., Frydendall, J. and Jorgensen, J. B. (2012), "Oil Reservoir Production Optimization 
using Single Shooting and ESDIRK Methods", in 291 (ed.), Proceedings of the 2012 IFAC Workshop on Automatic Control in Offshore Oil and Gas Production, May 31 - June 1, 2012, Norwegian University of Science and Technology, Trondheim, pp. 286-291.

[18] Foss, B. and Jensen,J. P. (2011), "Performance Analysis for Closed-Loop Reservoir Management", SPE Journal, vol. 16, no. 1, pp. pp. 183-pp. 183-190.

[19] Jansen, J. D., Bosgra, O. H. and Van den Hof, P. M. J. (2008), "Model-Based Control of Multiphase Flow in Subsurface Oil Reservoirs", Journal of Process Control, vol. 18, no. 9, pp. 846-855.

[20] Brouwer, D.R., NÃ'vdal, G., Jansen, J.D., Vefring, E.H. and van Kruijsdijk, C.P.J.W., ( 2004), Improved Reservoir Management Through Optimal Control and Continuous Model Updating, Society of Petroleum Engineers, Houston, Texas.

[21] Kowalska, K. and Mohrenschildt, M. v. (2011), "An Approach to Variable Time Receding Horizon Control", Optimal Control Applications and Methods, vol. 33, pp. 401-414.

[22] Goodwin, G. C., Seron, M. M. and Middleton, R. H. (2005), "Receding Horizon Control Applied to Optimal Mine Planning", Preprint Subimitted to Automatica.

[23] Jansen, J., Brouwer, R. and Douma, S. G. (2009), "Closed Loop Reservoir Management", SPE Reservoir Simulation Symposium, 2-4 February 2009, Society of Petroleum Engineers, The Woodlands, Texas. 